\title{
Erythrocyturia and proteinuria conversion in post-streptococcal acute glomerulonephritis
}

\author{
I Ketut Suarta
}

\begin{abstract}
Background Acute glomerulonephritis (AGN) is a sudden onset of macroscopic hematuria and edema. The chronic post-streptococcal acute glomerulonephritis (PSAGN) can be predicted if microscopic hematuria, proteinuria, and low serum complement C3 level are present for a period exceeding six months after initial onset of illness. It is prudent to follow the course of PSAGN until proteinuria normalizes and microhematuria disappears in urinalysis.

Objective To acquire the time of erythrocyturia and proteinuria conversion in post-streptococcal acute glomerulonephritis (PSAGN) among children.

Methods A retrospective cohort study on children with PSAGN was conducted in the Pediatric Outpatient Clinic and Ward at Sanglah Hospital, Denpasar, Bali from January 2001-December 2003. All subjects were recorded for clinical and laboratory signs, including initial symptoms, history of previous streptococcal infections, blood pressure, complete blood count, serum albumin, ASTO titer, complement C3 level, BUN, and serum creatinin. Erythrocyturia and proteinuria follow-ups were done by recording urinalysis findings for a six-month period after initial onset.

Results Thirty subjects 21 boys and 9 girls, mean age 8.7 (SD 2.5) years] with PSAGN were enrolled in the study, 16 had hematuria with edema. Twenty-one subjects had the history of upper respiratory tract infections and 9 with skin infections. Mean systolic blood pressure was 141.3 (SD 21.8) $\mathrm{mmHg}$, mean diastolic blood pressure was 90.8 (SD 16.3) $\mathrm{mmHg}$, mean ASTO titer was 1103.3 (SD 686.1) IU/ml, and mean complement C3 level was 42.2 (SD 15.5) mg/dl. Urinalysis monitoring showed 2/30, 9/30, 12/30, 22/30, 27/30 subjects had conversion hematuria at the first, second, third, fourth, and fifth month, respectively. Median duration of erythrocyturia conversion was 4.0 months (95\% Cl: 3.53-4.47). There were 11/30, 18/30, 21/30, 23/30, 25/30, 26/30 subjects with proteinuria conversion at the first, second, third, fourth, fifth, and sixth month, respectively. Median duration of proteinuria conversion was 2.0 months (95\% Cl: 1.25-2.75).

Conclusion Three out of 30 children remained with persistent hematuria and 4 of 30 remained with persistent proteinuria [Pediatr Indones 2006;46:71-76].
\end{abstract}

Keywords: Post-streptococcal acute glomerulonephritis, erythrocyturia, proteinuria,

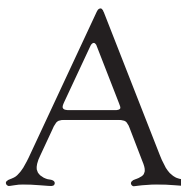

cute glomerulonephritis (AGN) is a syndrome characterized by the abrupt onset of macroscopic hematuria and edema. The majority of AGN instances appear to be post-infectious, and a number of bacterial and viral infections have been etiologically incriminated. The most commonly recognized is group A, â-hemolytic streptococcus infections, ${ }^{1}$ therefore, the term used in this report is post-streptococcal acute glomerulonephritis (PSAGN).

Certain nephritogenic strains of streptococci have been associated with PSAGN. The more common sporadic variety of PSAGN is the type 12 streptococcal infection of the pharynx. Epidemics of the disorder have been linked to several strains causing either throat or skin infections., ${ }^{2,3}$

PSAGN predominantly affects children between the ages of 2-10 years, with a slight predominance in males. ${ }^{2}$ Sardjito, ${ }^{4}$ in a multi-center study, found that the incidence of PSAGN predominantly occurrs in males with a male to female

From the Nephrology Division, Department of Child Health, Medical School, Udayana University, Sanglah Hospital, Denpasar, Bali, Indonesia.

Reprint requests to: I Ketut Suarta, MD, Nephrology Division, Department of Child Health, Medical School, Udayana University, Sanglah Hospital, Jl. Nias, Denpasar,Bali, Indonesia. Tel. 62-361-227911/ 227915; Fax. 62-361-244038. 
ratio of 1.3:1, and most commonly occurrs between the ages of $6-8$ years.

Typically, children with PSAGN present a sudden onset of painless gross hematuria and some edema. Hypertension is a general feature of PSAGN and may lead to hypertensive encephalopathy. The laboratory findings of PSAGN include increased ASTO titer and decreased serum complement C3. Urinalysis, in most cases, shows hematuria, proteinuria, and abnormal sediment including erythrocyte cast. ${ }^{1-3}$ In adult, $15 \%$ to $30 \%$ of PSAGN progresses to a chronic state while an estimation in children is ranged approximately from $5-10 \% .1,5$ The development of chronic PSAGN can be predicted if the microscopic hematuria, proteinuria, and low serum complement C3 level are present for a period exceeding six months after initial onset of illness. It is prudent to follow-up PSAGN patients until proteinuria normalizes and microhematuria has disappears in urinalysis. ${ }^{6}$

The aim of this study is to acquire the time of erythrocyturia and proteinuria conversion among PSAGN children.

\section{Methods}

A cohort retrospective study on children with PSAGN was conducted in the Pediatric Outpatient Clinic and Ward, Sanglah Hospital, Denpasar, Bali, from January 2001-December 2003. Data were collected from medical records.

All subjects who fulfilled the PSAGN criteria were recruited. The diagnosis of PSAGN was defined by the presence of edema and hematuria, ASTO titer $>200 \mathrm{IU} / \mathrm{ml}$, C3 level $<60 \mathrm{mg} / \mathrm{dl}$, erythrocyturia $>5 /$ high power field. Subjects were excluded from the study if data were incomplete.

All patients who met the criteria were recorded for clinical signs and laboratory findings, including initial symptoms, history of previous streptococcal infections, blood pressure, complete blood count, serum albumin, ASTO titer, complement C3 serum, BUN, and serum creatinin level. Follow-up for erythrocyturia and proteinuria was done by recorded urinalysis findings for a six-month period after initial onset of illness. Urinalysis examination for erythrocyturia and proteinuria was performed every month. The first urinalysis was done one month after the diagnosis was established (at first visit for follow-up).

Erythrocyturia was considered positive if the number of erythrocyte was $\geq 5$ /high power field in centrifuged urine, using light microscope. ${ }^{7}$ Proteinuria was considered positive if the amount of protein in urine was $>+1$ or $>30 \mathrm{mg} / \mathrm{dl}$, using semi-quantitative Combur-test dipstick.

Data were analyzed using SPSS 11.5 for Windows.

\section{Results}

During the study period, there were 34 children with PSAGN, 30 of them were enrolled, while 4 were excluded due to incomplete data. The mean age was 8.7 (SD 2.5) years. Male to female ratio was $2.3: 1$. The mean systolic blood pressure was 141.3 (SD 21.8) $\mathrm{mmHg}$, while mean diastolic blood pressure was 90.8 (SD 16.3) $\mathrm{mmHg}$. Characteristics of subjects are summarized on Table 1.

Figure 1 shows that 2/30, 9/30,12/30, 22/30, $27 / 30$ subjects had erythrocyturia conversion at the first, second, third, fourth, and fifth month, respec-

Table 1. Characteristics of Subjects

\begin{tabular}{|c|c|c|}
\hline Characteristics & Frequency & Mean (SD) \\
\hline Age (years) & & $8.7(2.5)$ \\
\hline \multicolumn{3}{|l|}{ Sex } \\
\hline Male & 21 & \\
\hline Female & 9 & \\
\hline \multicolumn{3}{|l|}{ Initial symptoms } \\
\hline Macroscopic hematuria & 6 & \\
\hline Edema & 8 & \\
\hline Hematuria + edema & 16 & \\
\hline $\begin{array}{l}\text { History of upper respiratory tract } \\
\text { infection }\end{array}$ & 21 & \\
\hline History of skin infection & 9 & \\
\hline \multicolumn{3}{|l|}{ Blood pressure } \\
\hline Systolic (mmHg) & & $141.3(21.8)$ \\
\hline Diastolic (mmHg) & & $90.8(16.3)$ \\
\hline \multicolumn{3}{|l|}{ Laboratory } \\
\hline White blood cell $(\mathrm{k} / \mu \mathrm{l})$ & & $11.5(3.7)$ \\
\hline Hemogolobin (g/dl) & & $10.5(1.2)$ \\
\hline Serum albumin (g/dl) & & $3.1(0.4)$ \\
\hline ASTO titer (IU/ml) & & $1103.3(686.1)$ \\
\hline Complement C3 level (mg/dl) & & $42.2(15.5)$ \\
\hline BUN (mg/dl) & & $31.9(27.9)$ \\
\hline Serum creatinin $(\mathrm{mg} / \mathrm{dl})$ & & $1.3(1.4)$ \\
\hline
\end{tabular}




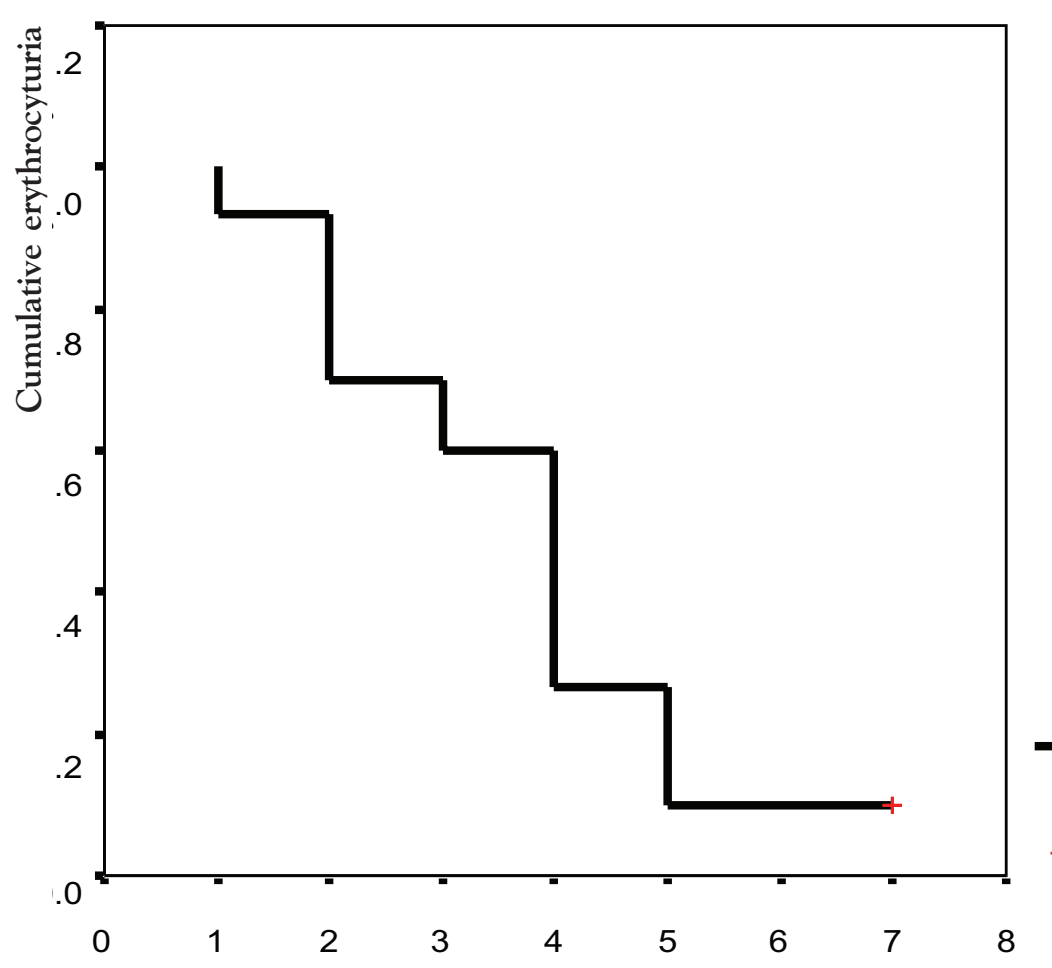

Erythrocyturia conversion (month)

Figure 1. Kaplan-MeIer CURVE of ERYTHROCYTURIA CONVERSION DURING A SIX-MONTHS FOLLOW-UP OF PSAGN SUBJECTS

tively. Only 3 out of 30 children remained to have persistent hematuria over a six-month period of follow-up. Median duration of erythrocyturia conversion was 4.0 months (95\% CI: 3.53-4.47).

The proteinuria conversion was also presented in a Kaplan-Meier curve (Figure 2). Figure 2 shows that 11/30,18/30,21/30,23/30, 25/ $30,26 / 30$ subjects had proteinuria conversion at the first, second, third, fourth, fifth, and sixth month, respectively. Only 4 out 30 children remained to have persistent proteinuria over a sixmonth period of follow-up. Median duration of proteinuria conversion was 2.0 months $(95 \% \mathrm{CI}$ : $1.25-2.75)$.

\section{Discussion}

The true incidence of PSAGN is unknown. Due to the large number of affected children with mild and often unrecognized manifestations, it is undoubtedly much higher than statistics would indicate. ${ }^{1}$ The highest of incidence is reported in early school years, and the disease is clinically uncommon in children under 3 years of age and most commonly occurs between 6-8 years of age. It is reported that the male to female ratio of PSAGN is $2: 1,8$ while Sardjito 4 reported 1.3:1, and Pardede et al ${ }^{9}$ reported 1.7:1. In our study, the mean age of children with PSAGN was $8.7 \pm(\mathrm{SD} 2.5)$ years and ratio of male to female was 2.3:1.

Clinical presentations of PSAGN are widely vary. Classic features present a history of upper respiratory tract infection approximately two weeks prior to the onset of swelling. In PSAGN with previous skin infection, the interval between onset of infection and onset of nephritis maybe difficult to determine. ${ }^{10}$ Our study found 21 subjects with history of upper respiratory tract infection and 9 subjects with skin infection. However, we did not investigate the duration between infection and onset of PSAGN. 


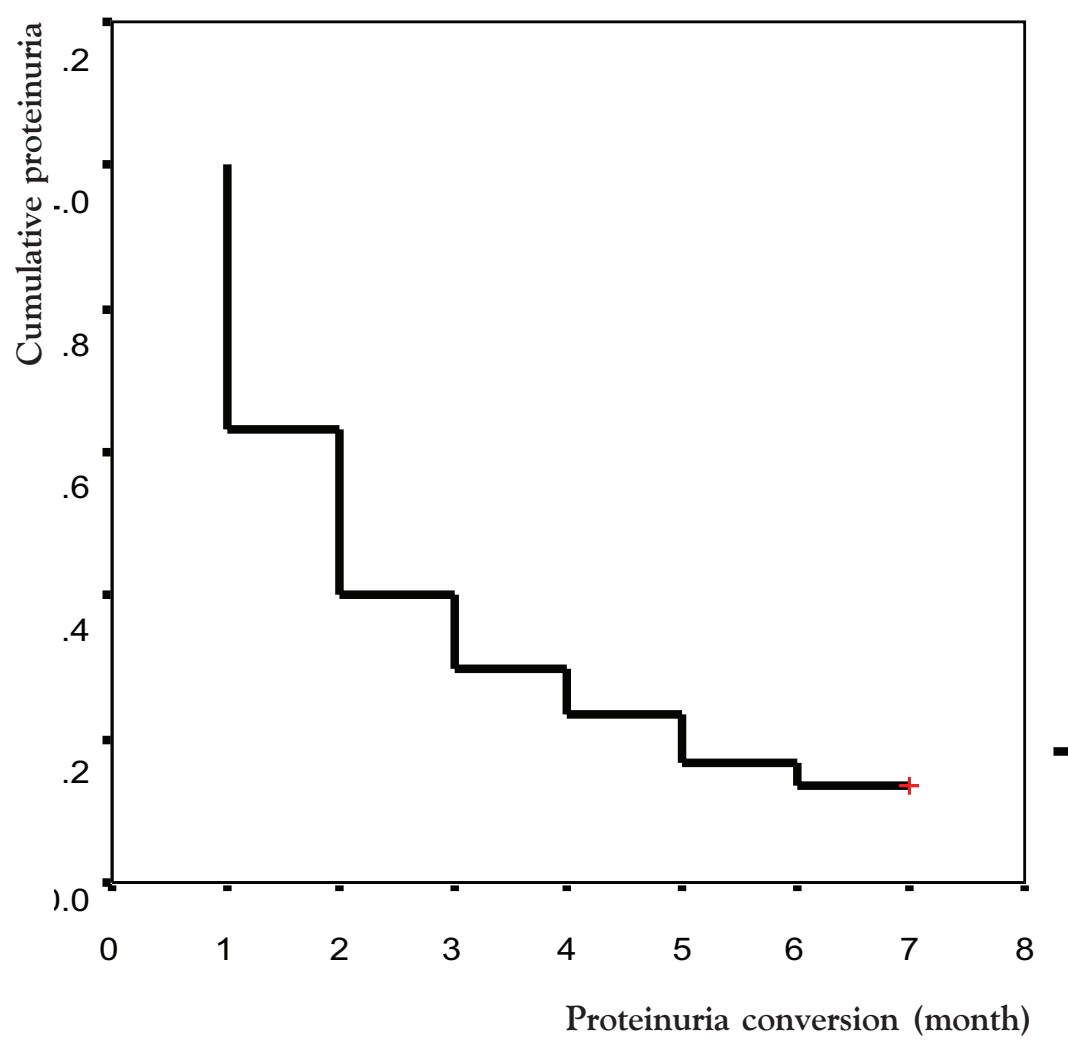

Figure 2. KapLAN-MEIER CURVE OF PROTEINURIA CONVERSION DURING A SIXMONTH FOLLOW-UP OF PSAGN SUBJECTS

Gross hematuria was present in 30-50\% of hospitalized patients, while hypertension was present in the majority of hospitalized patients. The pathogenesis of hypertension is unknown, yet it is possibly multifactorial and related only in part to volume expansion. The degree of hypertension is variable and not proportional to the degree of edema. ${ }^{10}$ In our study, the most common initial symptoms was gross hematuria combined with edema, which occurred in 16 cases. Mean systolic and diastolic blood pressure were 141.3 (SD 21.8) $\mathrm{mmHg}$ and 90.8 (SD 16.3) $\mathrm{mmHg}$, respectively.

Direct or indirect evidence of a preceding streptococcal infection should be sought in all children presenting with acute nephritis, regardless of history. Negative culture may be seen if adequate antibiotic treatment has been received, or if the interval between initial infection and onset of clinical nephritis has been prolonged. If direct culture evidence of streptococcal infection is lacking, serological tests to demonstrate an immune response to streptococ- cal antigen should be pursued. The serological test may reveal raised antistreptolysin antibody $\mathrm{O}$ (ASTO) titer, but its absence does not exclude the diagnosis as many nephritogenic strains do not produce streptolysin. Elevation of ASTO titer will be seen within 10-14 days following a streptococcal infection in most patients, and will remain elevated for several months. ${ }^{3,8,10}$ An important and very consistent finding in PSAGN is a lower range of the third complement (C3). Serum C3 levels will be below normal at onset of symptoms in $80-90 \%$ of patients. Low C3 levels with normal C4 levels (due to alternative pathway activation) are seen acutely, but should have returned to normal limit within two months. ${ }^{8,10,11}$ In this study, the culture from throat swab or skin was not performed in all subjects. From serological investigation, we found mean ASTO titer was $>1000 \mathrm{IU} / \mathrm{ml}$ while serum C3 level was low. Unfortunately, serum C3 level only checked once at the admission, so we could not see the fluctuation and time to return to normal limit. 
In general, the acute phase of illness of PSAGN with its most severe manifestations lasts for 2-3 weeks. Urinary findings may take much longer to resolve. While gross hematuria is likely to be clear by 3 weeks from onset of illness, microscopic hematuria may persist for up to 6 months, and often accompanied by proteinuria for 6 months. ${ }^{10}$ Previous study by Ninik et $a l^{12}$ showed persistent microhematuria was found in $8.9 \%$ and persistent proteinuria was in $1.7 \%$ of children with PSAGN during an 11 years period of follow-up. Sarkissian et al..$^{5}$ reported that complete remission was noted at follow-up in 78\% children after three months and in $97 \%$ children examined in one year. White et al. ${ }^{13}$ in their study found hematuria was present in 21\% of the PSAGN patients in a 14 year period of follow-up. Our study showed persistent erythrocyturia in $10 \%$ and persistent proteinuria in $13 \%$ after a six month period of follow-up.

The frequency of follow-up examination for patients with PSAGN will depend on a great extent of the individual patient and the severity of the disease. Measurement of blood pressure and examination of urine for erythrocyte and protein will be the best means to evaluate recovery. After a 6-month period since the initial symptoms appearance, if hematuria and proteinuria have resolved, a yearly urinalysis and blood pressure determination is sufficient. ${ }^{10}$

In several conditions, renal biopsy should be considered, i.e. renal function is severely impaired particularly if etiology is uncertain; atypical presentation such as anuria, nephrotic syndrome presentation; delayed resolution with persistent hypertension, azotemia, and gross hematuria after 3 weeks, persistently low $\mathrm{C} 3$ level at 6 weeks, persistent proteinuria after 6 months, persistent hematuria after 12 months. ${ }^{10}$

A controversy exists concerning the long-term prognosis of PSAGN. While it is generally agreed that the outcome is more favorable in children than in adults, follow-up studies reported on the incidence of impaired renal function that range from less than 1\% to as high as $20-30 \%$. Overall, it can probably be said with a reasonable degree of assurance that greater than $90 \%$ of children with PSAGN should be expected to recover, without significant alteration in renal function. Of children with PSAGN, a small minority of those hospitalized, $0.5-2.0 \%$ may have a rapid progression of their renal disease, with reaching end-stage within weeks to months. ${ }^{5,10}$
The limitations in this study were the follow-up period only six months; it would be more useful to extend monitoring up to one year. The best means to evaluate recovery were by measurement of blood pressure, examination of urine for erythrocyte and protein, and monitoring serum complement C3 level. In our study, we only did follow-up by looking at erythrocyturia and proteinuria conversion.

In conclusion, only 3 out of 30 children with PSAGN still had persistent hematuria and 4 out of 30 children still had persistent proteinuria, over a sixmonth period of follow-up. These children need more attention and longer follow-up period.

\section{References}

1. Brouhard BH, Travis LB. Acute postinfectious glomerulonephritis. In: Edelmann CM, editor. Pediatric kidney disease. 2nd ed. Boston: Little, Brown and Co; 1992. p. 1199-215.

2. Hricik DE, Chung-Park M, Sedor JR. Glomerulonephritis. N Engl J Med 1998;339:888-99.

3. Cunningham MW. Pathogenesis of group A Streptococcal infection. Clin Microbiol Rev 2000;13(3):470-511.

4. Sardjito. Profile PSAGN in Indonesian children: a multi center study. Proceedings of the $1^{\text {st }}$ Annual Pediatric Meeting; 2001 June 25-27; Palembang, Indonesia.

5. Sarkissian A, Papazian M, Azatian G. An epidemic of acute postinfectious glomerulonephritis in Armenia. Arch Dis Child 1997;77:342-4.

6. Makker SP. Glomerular diseases. In: Kher KK, Makker SP, editors. Clinical pediatric nephrology. 2nd ed. New York: McGraw-Hill Inc; 1992. p. 212-20.

7. Sokolosky MC. Hematuria. Emerg Med Clin North Am 2001;19(3):621-32.

8. Vinen CS, Oliveira DBG. Acute Glomerulonephritis. Postgrad Med J 2003;79:206-13.

9. Pardede SO, Trihono PP. Clinical features of acute glomerulonephritis in children. Proceedings of the $12^{\text {th }}$ National Child Health Congress; 2002 June 30-July 4; Bali, Indonesia.

10. Travis LB, Kalia A. Acute nephritic syndrome. In: Postlethwaite RJ, editor. Clinical paediatric nephrology. 2nd ed. Oxford: Butterworth-Heinemann, 1994. p. 201-9.

11. Mason PD. Fortnightly review: glomerulonephritis diagnosis and treatment. Br Med J 1994;309:1557-63. 


\section{Paediatrica Indonesiana}

12. Ninik AS. Long-term prognosis of post infectious glomerulonephritis after an acute episode. Proceedings of the 12th National Child Health Congress; 2002 June 30-July 4; Bali, Indonesia.
13. White AV, Hoy WE, McCredie DA. Childhood poststreptococcal glomerulonephritis as a risk factor for chronic renal disease in later life. Med J Aust 2001;174:492-6. 